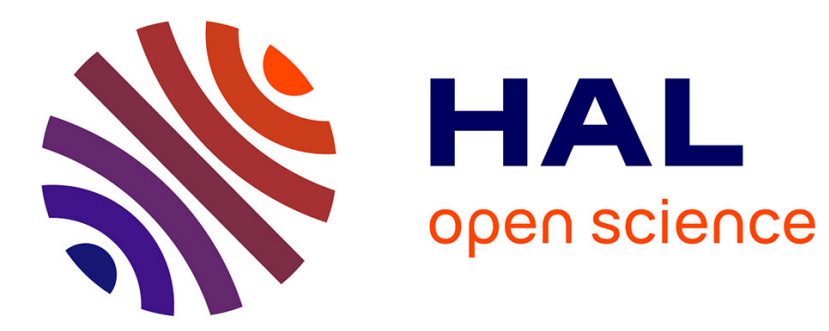

\title{
Réappropriation foncière dans les campagnes d'Europe centrale
}

\author{
Marie-Claude Maurel
}

\section{To cite this version:}

Marie-Claude Maurel. Réappropriation foncière dans les campagnes d'Europe centrale. Gérard Chouquer; Marie-Claude Maurel. Les mutations récentes du foncier et des agricultures en Europe, Presses universitaires de Franche-Comté, pp.113-136, 2019, Les Cahiers de la MSHE, 978-2-84867-641-8. hal03248669

\section{HAL Id: hal-03248669 \\ https://univ-fcomte.hal.science/hal-03248669}

Submitted on 3 Jun 2021

HAL is a multi-disciplinary open access archive for the deposit and dissemination of scientific research documents, whether they are published or not. The documents may come from teaching and research institutions in France or abroad, or from public or private research centers.
L'archive ouverte pluridisciplinaire HAL, est destinée au dépôt et à la diffusion de documents scientifiques de niveau recherche, publiés ou non, émanant des établissements d'enseignement et de recherche français ou étrangers, des laboratoires publics ou privés. 


\section{Les mutations récentes}

\section{du foncier et des agricultures en Europe}

sous la direction de

Gérard CHOUQUER

et Marie-Claude MAUREL



Presses universitaires de Franche-Comté

Presses universitaires Sun Yat-sen de Guangzhou 
Illustration de couverture :

Vue aérienne des champs agricoles

Source : (C) 123RF/Peter Gudella 


\section{LES MUTATIONS RÉCENTES DU FONCIER ET DES AGRICULTURES EN EUROPE}




\section{PRESSES UNIVERSITAIRES DE FRANCHE-COMTÉ}

\section{$\mathrm{N}^{\circ} 1445$}

\section{Collection « LES CAHIERS DE LA MSHE LEDOUX » dirigée par Philippe Barral \\ $n^{\circ} 35$}

Série "Normes et pratiques foncières et agricoles dans le monde » éditée en version originale chinoise par les Presses universitaires Sun Yat-sen de Canton, puis en version française par les Presses universitaires de Franche-Comté Responsable : Gérard Chouquer

$$
\mathrm{n}^{\circ} 1
$$

\section{MSHE Claude Nicolas Ledoux, USR 3124 \\ 30-32, rue Mégevand \\ 25030 Besançon cedex}

\footnotetext{
(C) PRESSES UNIVERSITAIRES DE FRANCHE-COMTÉ

ET PRESSES UNIVERSITAIRES SUN YAT-SEN DE CANTON - 2018

Mise en page et suivi éditorial de la version française : Marie Gillet
}

ISBN : 978-2-84867-641-8 - EAN : 9782848676418

ISSN : 1771-8988 


\title{
LES MUTATIONS RÉCENTES DU FONCIER ET DES AGRICULTURES EN EUROPE
}

\author{
sous la direction de \\ Gérard Chouquer et Marie-Claude Maurel
}

\begin{abstract}
auteurs :
Guihem Anzalone, Gilles Bazin, Hubert Bosse-Platière,

Pascal Chevalier, Gérard Chouquer, Hubert Cochet, Sophie Devienne, Clémence Guimont, Bertrand Hervieu, Guillaume Lacquement, Marie-Claude Maured, Geneviève NGuyen, François Purseigle, Aurélie Trouvé, Bruno Villalba
\end{abstract}

Ouvrage publié avec le concours des Pressses universitaires Sun Yat-sen de Canton (Chine) 
La création de cette collection est une opportunité due à la volonté des Presses de l'Université Sun Yat-sen de Guangzhou (province de Guangdong). Grâce à la présence d'un important département de français dans cette université, les Presses universitaires Sun Yat-sen ont choisi de s'investir dans la mise à disposition du public chinois de traductions d'ouvrages originaux de langue française sur les questions foncières, agricoles et environnementales, qui permettent à la recherche et à l'enseignement universitaire chinois de disposer d'ouvrages équilibrant les traductions d'ouvrages anglo-saxons dont ils disposent déjà.

Lors de la mise en route, avant le lancement de cette collection, de la traduction de deux ouvrages déjà publiés (Bertrand Hervieu et François Purseigle, 2013, Sociologie des mondes agricoles, Paris, Armand Colin; Gérard Chouquer, 2012, Terres porteuses, Arles, Actes Sud), il a été décidé d'un commun accord de faire porter l'effort sur la publication d'ouvrages inédits dont la version originale sera publiée dans une collection nouvelle en Chine puis, quasi simultanément, en version française dans une série également nouvelle de la collection des "Cahiers de la Maison des sciences de l'homme et de l'environnement » de Besançon, en raison de la cession de droits consentie par les Presses universitaires Sun Yat-sen aux Presses universitaires de Franche-Comté.

L'association France internationale pour l'expertise foncière, FIEF (Ordre des géomètres-experts) a manifesté son intérêt pour cette initiative et en a facilité la réalisation, notamment en apportant un concours juridique à la rédaction des contrats et en proposant son réseau d'experts fonciers.

Les Presses universitaires Sun Yat-sen de Guangzhou et les Presses universitaires de Franche-Comté, ont confié la direction de la collection ou de la série à Gérard Chouquer (ancien directeur de recherches au CNRS; expert foncier et secrétaire de FIEF), et ont sollicité Marie-Claude Maurel (ancienne directrice du département Sciences de l'homme et de la société du CNRS, directrice d'études à l'EHESS) pour qu'elle assure la suppléance de cette direction et apporte son conseil à la conduite du projet. 


\section{Remerciements}

Les Presses universitaires Sun Yat-sen de Guangzhou et les Presses universitaires de Franche-Comté remercient France internationale pour l'expertise foncière (Ordre des géomètres-experts, 40 avenue Hoche, 75008 Paris, France), pour l'aide proposée lors la réalisation de cet ouvrage »

Gérard Chouquer et Marie-Claude Maurel remercient Monsieur Xu Jing, directeur des Presses universitaires Sun Yat-sen de Guangzhou et Madame Zeng Xiaoyang, directrice du département de français de cette université, pour l'accueil qu'ils ont réservé à ce projet et pour l'extrême qualité des échanges lors du suivi de cette réalisation.

Ils remercient également Jean-Paul Barrière, directeur des Presses universitaires de Franche-Comté, et Philippe Barral, directeur de la Maison des sciences de l'homme et de l'environnement Claude Nicolas Ledoux (Besançon), pour leur accueil et leurs encouragements. 


\title{
Chapitre 5
}

\section{Réappropriation foncière dans les campagnes d'Europe centrale}

\author{
Marie-Claude MAUREL
}

Dans les pays d'Europe centrale, les structures d'exploitation agricole issues du démantèlement du collectivisme agraire présentent une grande diversité de formes sociales de production tant du point de vue des dimensions que des logiques de fonctionnement. Petites et très grandes exploitations, exploitations familiales et exploitations sociétaires coexistent en proportions variées selon les régions. Si les catégories statistiques et normatives usuelles permettent de repérer la singularité des profils structurels de ces agricultures, elles se révèlent impropres à appréhender leurs trajectoires de transformation dans le contexte du changement de système économique et social. Comment naissent, survivent ou disparaissent les divers types d'exploitations lorsque l'environnement institutionnel est radicalement modifié ?

Cette interrogation invite, dans une première partie, à retracer les modalités de privatisation de la terre et du capital mises en œuvre et leurs effets sur la transformation des structures postcollectivistes. La diversité des trajectoires de transformation des structures d'exploitation fait l'objet d'une deuxième partie qui analyse en quoi les évolutions structurelles propres à ces agricultures diffèrent des structures d'exploitation des anciens États membres de l'Union européenne* (UE). Du fait de leur intégration dans cette dernière, les agricultures des nouveaux États membres* (NEM) sont devenues bénéficiaires des aides directes et des mesures de soutien de la politique agricole commune* (PAC) et en ressentent l'impact sur leurs dynamiques de transformation. Retracés dans une dernière partie, les effets des politiques agricoles nationales et européennes se révèlent diversement appropriés aux particularités du contexte socioéconomique de chacun de ces pays. À l'opposé de la convergence initialement attendue, une forte hétérogénéité des formes sociales de production reste la marque distinctive des agricultures d'Europe centrale. 


\section{La mutation postcollectiviste des structures agraires}

Engagée au lendemain de la fin des régimes communistes, la transition vers l'économie de marché a entraîné de profondes mutations des structures agraires du fait du rétablissement de la propriété privée sur la terre et les moyens de production, et d'un changement du mode de régulation économique. La décollectivisation* doit s'entendre comme un processus global s'appliquant aux trois facteurs de production essentiels en agriculture - la terre, le capital et le travail - opérant dans le contexte d'un changement de système économique et social lié au passage au marché. Les transformations ont été engagées sans que, faute de consensus politique, le choix d'un modèle agricole de substitution ait pu être déterminé. Priorité a été donnée par les gouvernements postcommunistes au rétablissement des droits de propriété sur la terre et le capital d'exploitation ${ }^{1}$, ainsi qu'au changement de statut juridique des anciennes exploitations collectives.

\section{D'un usage collectif à un usage privé des moyens de production}

\section{Les dispositifs de transfert des droits de propriété}

La réhabilitation de la propriété privée a placé le législateur devant la responsabilité de définir les principes d'une redistribution des droits sur la terre et le capital de production. Les dispositifs juridiques ont combiné, dans des proportions variables selon les pays, plusieurs approches : la restitution (et/ou la compensation) des biens confisqués aux anciens propriétaires, l'attribution de nouveaux droits sur la terre aux travailleurs agricoles (ou aux ménages ruraux), la mise en vente des terres du fonds foncier d'État aux exploitants agricoles. Ces diverses modalités concernaient des catégories d'ayants droit aux caractéristiques bien distinctes : les anciens propriétaires, en majorité âgés, et leurs descendants, le plus souvent partis en ville, les membres des coopératives et les employés des fermes d'État, les spécialistes et les dirigeants de ces grandes exploitations. La réappropriation d'un bien foncier ne présentait ni la même signification ni la même valeur pour chacune de ces catégories. Quelles que soient les modalités mises en œuvre, ces procédures ont eu pour effet de recréer, dans la plupart de ces pays, une masse de petits propriétaires fonciers dont l'intérêt pour l'usage agricole de la terre était extrêmement variable.

Les dispositifs de privatisation du capital d'exploitation (bâtiments, cheptel et matériel) ont été intégrés aux procédures de transformation juridiques des coopératives de production, d'une part, des fermes d'État, d'autre part. Dans le premier cas, la répartition des parts de capital, après estimation de leur valeur, s'est opérée au bénéfice des ayants droit en fonction de leur apport initial en biens et/ou de leur contribution en travail. La conversion de leur statut juridique en associations agricoles ou en coopératives de propriétaires a suivi. Dans le second cas, la privatisation des biens s'est opérée sous le contrôle de l'État propriétaire ou de ses agences. Diverses formules de privatisation ont été appliquées : mise en gérance ou en location, mise en vente des terres, transformation en société de droit privé.

1. On entend par là le rétablissement d'un droit réel de propriété portant sur la totalité des droits, c'est-à-dire les trois catégories définies par l'usus, le fructus et l'abusus, au sens du droit latin. 


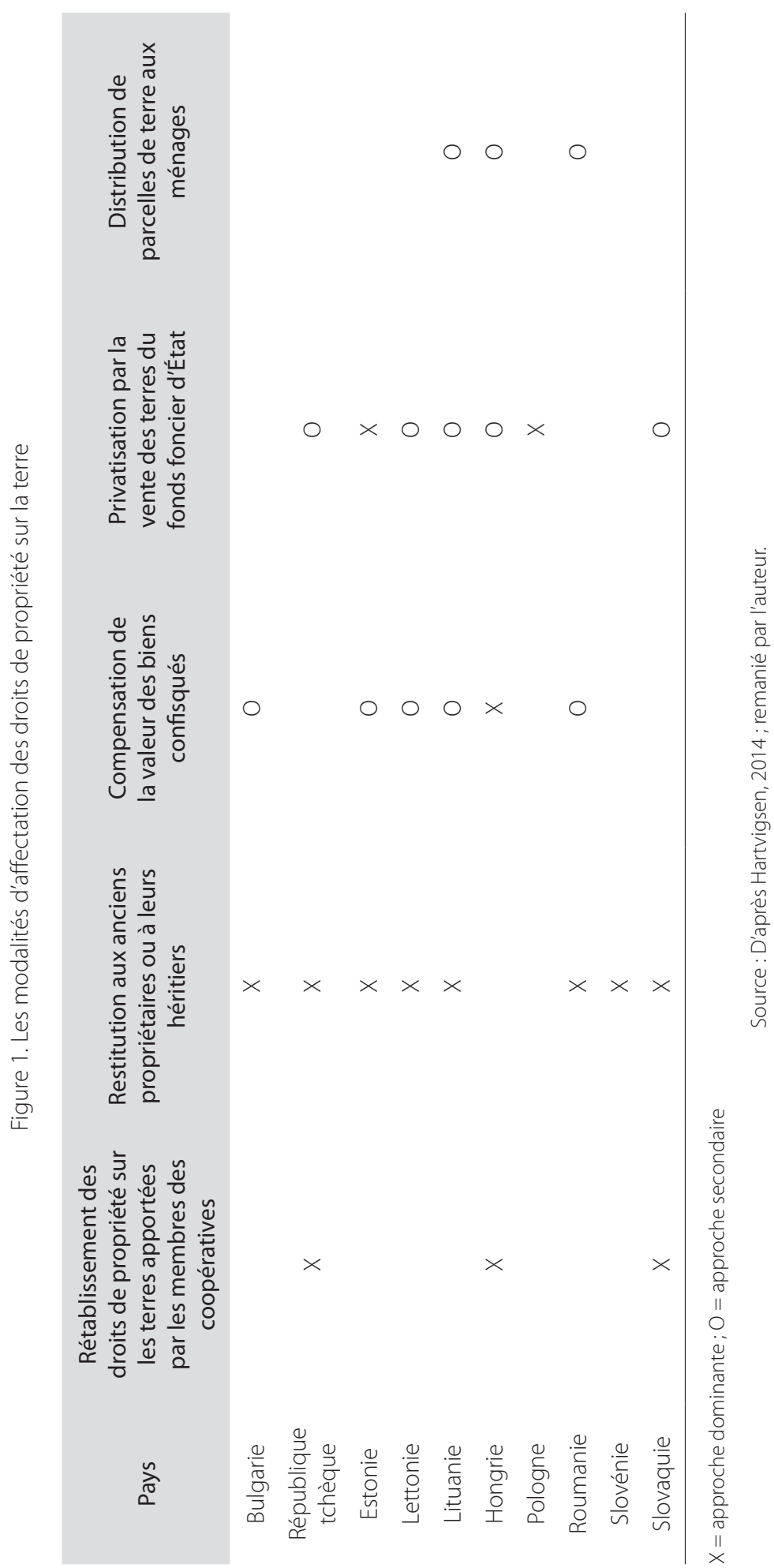




\section{Le rôle des acteurs sociaux dans la transformation}

Encadrées par les dispositifs législatifs adoptés par chaque État, les trajectoires de réappropriation de la terre et des moyens de production dépendaient avant tout de la prise d'initiative des acteurs sociaux, de leur détermination à s'engager dans un projet de reprise ou de création d'une exploitation agricole, en fonction de leur dotation initiale en facteurs de production. Une partie de ceux qui travaillaient dans les exploitations agricoles ne possédaient pas les compétences pour conduire de manière autonome une exploitation agricole. La stricte division du travail régissant l'agriculture collectiviste avait fait perdre aux travailleurs les moins qualifiés la possibilité d'acquérir les larges compétences nécessaires à l'organisation du procès de production. Quant aux anciens propriétaires qui avaient rompu le lien avec l'activité agricole, ils étaient dépourvus des savoir-faire indispensables. De manière générale, seul le groupe des spécialistes (techniciens, ingénieurs, vétérinaires) et celui des cadres dirigeants, disposaient des aptitudes managériales requises pour relever le défi de l'exploitation indépendante. Dans les conditions de sortie du système collectiviste, la recomposition des rapports de propriété s'est principalement opérée au bénéfice des acteurs les plus aptes à mobiliser les diverses formes de capital, social, économique et culturel. Libre cours a été donné aux acteurs sociaux les plus entreprenants pour se saisir des opportunités d'accession à la terre et aux moyens de production.

\section{Les effets du transfert :}

\section{fragmentation foncière et dissociation des facteurs de production}

Les procédures de distribution des droits de propriété sur la terre ont provoqué un processus de fragmentation foncière qui revêt plusieurs aspects. Là où la restitution aux anciens propriétaires a prévalu, les nouvelles structures de propriété présentent des similitudes avec celles qui existaient avant la collectivisation*. C'est le cas dans les États baltes où les réformes foncières de l'entre-deux-guerres avaient établi une paysannerie propriétaire de ses terres, détruite à partir de 1940 par l'occupation soviétique. En République tchèque et en Slovaquie, les règles de transmission aux héritiers sont venues ajouter leurs effets à ceux de la restitution pour accentuer la fragmentation de la propriété foncière. Tant que l'usage du sol reste concentré au profit de structures d'exploitation qui louent les terres auprès d'un grand nombre de petits propriétaires, ce morcellement de la propriété foncière n'a qu'un impact limité sur l'utilisation du sol. En Hongrie, dans le cadre de la procédure de compensation des biens, la mise en vente aux enchères d'une partie des terres auprès des porteurs de bons de compensation a permis que se constituent des exploitations individuelles de taille moyenne. Dans le même temps, l'attribution de parcelles aux membres des coopératives comme aux employés des fermes d'État a multiplié le nombre de petits propriétaires parcellaires. En Roumanie et en Bulgarie, la redistribution des terres s'est opérée par le retrait de parcelles à l'initiative des membres des coopératives, parfois même avant l'adoption des lois de restitution aux anciens propriétaires. Le morcellement foncier* affecte la propriété du sol ainsi que son usage. En Pologne et en Slovénie, où les petits paysans propriétaires avaient réussi à échapper à une collectivisation* totale, les structures agraires se caractérisent par un morcellement foncier plus ou moins accentué selon les régions.

Quels que soient leurs effets sur la propriété foncière, les dispositifs institutionnels encadrant le processus de décollectivisation ont dissocié les trois facteurs de production 
que sont la terre, le capital et le travail, auparavant assemblés sous une même direction dans le cadre des exploitations collectives.

\section{Des exploitations collectives aux nouvelles formes sociales de production}

La diversité des formes sociales de production résulte de la déconcentration* foncière et de la mutation des modes d'organisation du travail agricole. Les modalités de conversion des structures collectivistes ont été mises en œuvre sans réelle prise en considération des conditions de viabilité des exploitations successeurs. Pour qualifier ces formes sociales, on propose une catégorisation à visée interprétative tenant compte de l'affectation des droits de propriété sur la terre et sur le capital d'exploitation, ainsi que du mode d'articulation des rapports de propriété et des rapports de production caractérisant chacune de ces formes sociales. Cette approche s'applique aux exploitations qui ont pris la succession des structures collectivistes comme aux exploitations nouvellement installées. Nées d'une combinaison inédite des trois facteurs de production, certaines de ces formes sociales se sont révélées de type transitoire. Après quelques années, les néocoopératives de propriétaires, les entreprises publiques (issues de la conversion des fermes d'État) ont modifié leur statut juridique de manière à stabiliser le capital d'exploitation et à consolider le mode de gestion managériale. Par ailleurs, une partie des nouvelles exploitations individuelles établies sur les terres restituées ont échoué dans leur projet d'installation. De plus, la recomposition des structures agraires s'est déroulée dans le contexte de politiques économiques nationales qui ont beaucoup varié, notamment en termes de ciblage privilégié d'une catégorie d'exploitation par rapport aux autres. Chaque forme sociale de production peut être définie par un mode d'articulation de la terre, du capital et du travail qui lui est spécifique (fig. 2).

Leur filiation relève de mécanismes complexes de transmission, de reproduction et d'adaptation. Les grandes exploitations sont généralement issues de la privatisation des fermes d'État et de la conversion des coopératives de propriétaires. Souvent privatisées à l'initiative de leurs anciens cadres dirigeants, par des stratégies d'appropriation-contrôle de leur capital, elles fonctionnent majoritairement sous une forme sociétaire* (société par actions, société à responsabilité limitée), emploient des salariés et produisent exclusivement pour le marché. Ces grandes exploitations dont la taille moyenne est de l'ordre de quelques centaines à quelques milliers d'hectares cultivent une part prépondérante de la surface agricole utile ( $\mathrm{SAU}$ ) en Slovaquie, en République tchèque, en Bulgarie et en Estonie, et plus des deux cinquièmes en Hongrie et en Roumanie. Par leur haut niveau capitalistique, leur intégration au sein de grands groupes agroalimentaires, une partie de ces entreprises participent d'une agriculture de firme*.

La voie de la reconstitution d'une exploitation individuelle ou familiale a été empruntée par une minorité de ceux qui ont pu se réapproprier un bien foncier. L'opportunité de retirer des terres et des équipements des anciennes exploitations coopératives, la possibilité de bénéficier des restitutions ont permis à des agriculteurs de s'installer de manière indépendante. Cette agriculture de type individuel ou familial revêt diverses formes sociales qui se différencient par les dimensions, le niveau d'équipement, l'orientation de la production vers le marché et/ou l'autoconsommation. Renouant avec une logique familiale, fondée sur une articulation étroite entre la terre, le capital et le projet familial, les exploitations installées au lendemain de la décollectivisation* combinent un 


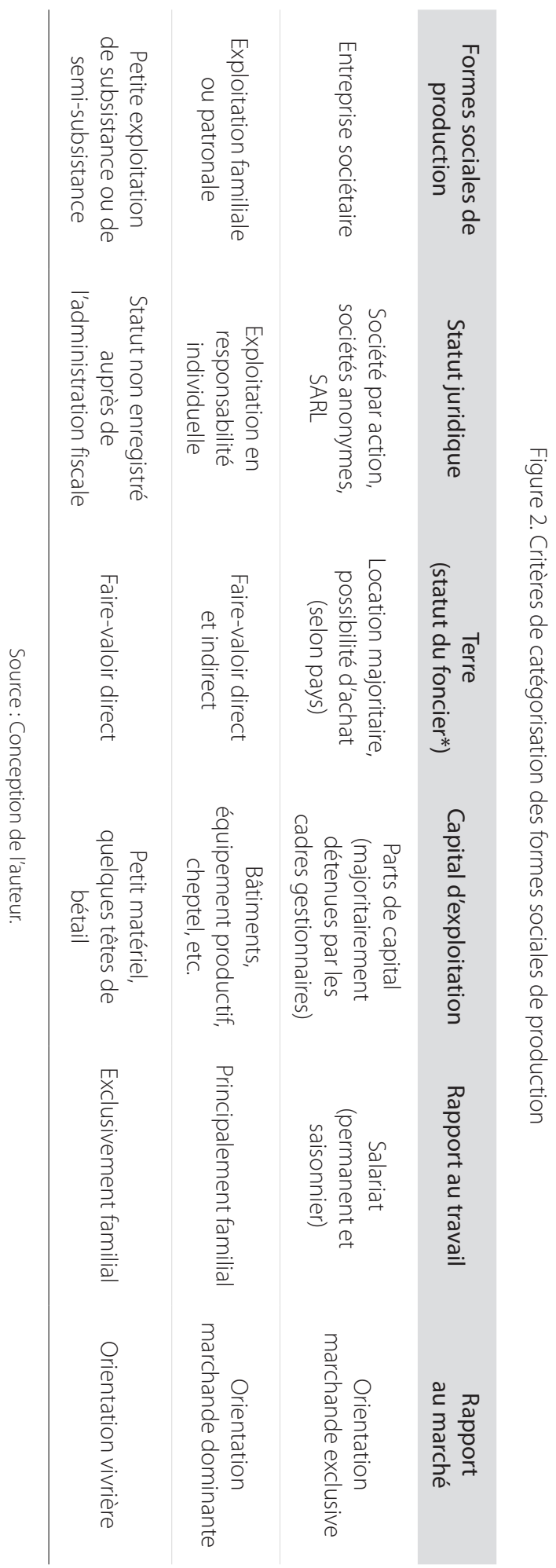




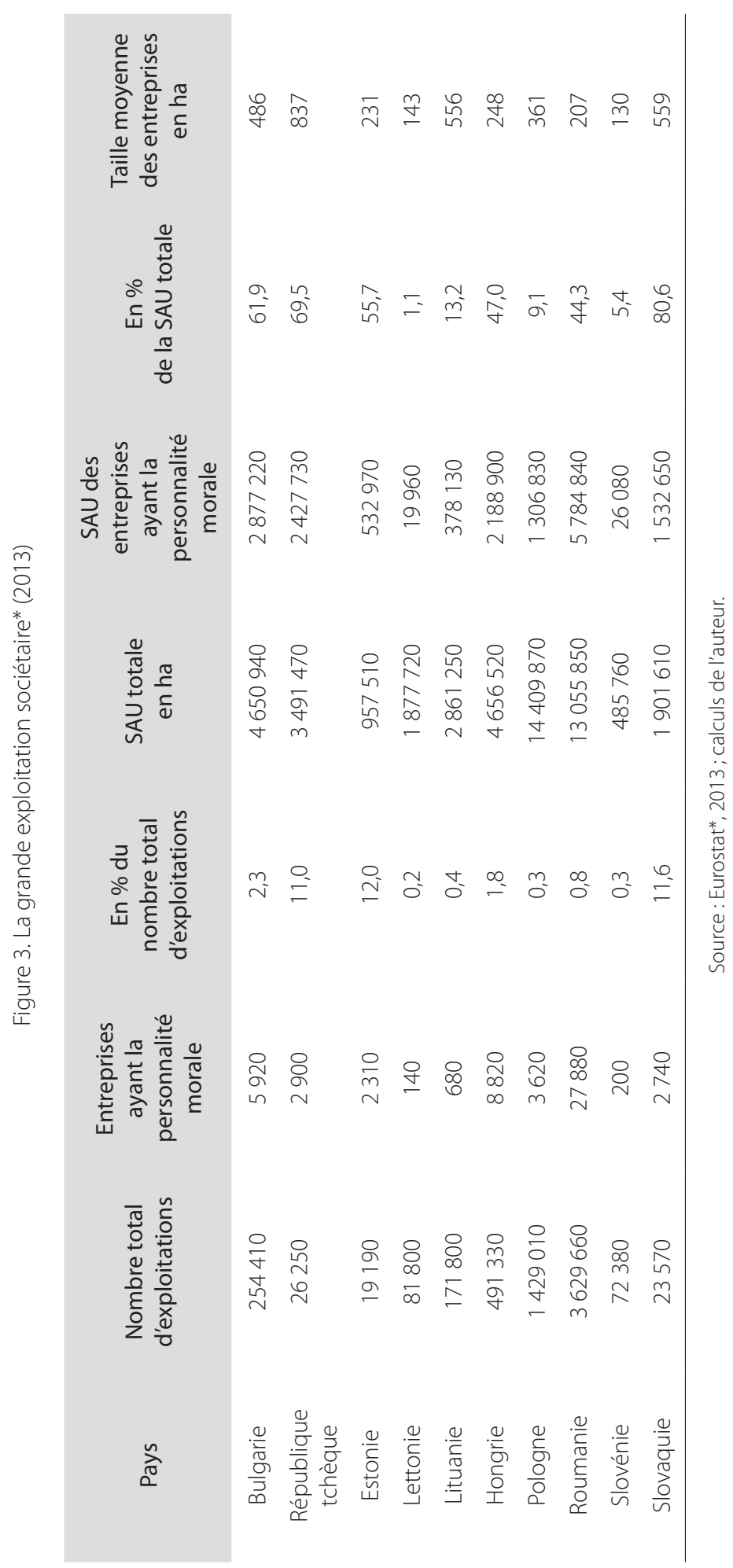


mode de faire-valoir direct avec des locations de terre afin d'élargir leur assise foncière. Dans les conditions incertaines qui ont présidé à la sortie du collectivisme, les difficultés rencontrées par les nouveaux exploitants pour accéder au crédit, bénéficier d'une assistance technique adéquate et écouler la production sur le marché ont pu entraîner l'abandon du projet initial. Une lente consolidation des exploitations moyennes familiales ou patronales s'esquisse à présent dans certains pays (Lituanie, Lettonie, Hongrie). Seules les unités relevant des catégories de taille supérieure à 20 ha et surtout au-delà de 50 ha, se trouvent en situation d'élargissement foncier, d'investissement et de modernisation. Dans leur grande majorité, ces exploitants cherchent à élargir leur assise foncière mais l'insuffisante fluidité du marché foncier constitue une entrave à leur développement.

Les plus petites exploitations ont pour origine les anciens lopins de l'économie auxiliaire attribués aux travailleurs agricoles pour leur usage propre (autoconsommation). Les politiques de redistribution des terres ont permis d'agrandir ces lopins (en Lituanie jusqu'à 3 ha). Les critères caractérisant ces microexploitations (superficie, taille économique, participation au marché) varient selon les pays. Leur statut juridique est souvent mal établi en raison de l'absence d'enregistrement de leur activité productive. La logique productive est principalement dictée par la satisfaction des besoins alimentaires du ménage. Ces microexploitations assurent la survie des catégories sociales les plus démunies (retraités, chômeurs) et jouent ainsi un rôle décisif d'amortisseur social. En fournissant un complément de revenu aux ménages pluriactifs comme aux retraités, elles remplissent une fonction sociale qui contribue à compenser la pauvreté en milieu rural. Cette agriculture de subsistance et de semi-subsistance est composée de sousgroupes d'exploitations, classées selon leur taille (moins de 2 ha, moins de 5 ha), et/ou la valeur de leur production standard (inférieure à 2000 euros, inférieure à 8000 euros). Leur importance, au moins en nombre d'exploitations, est considérable en Roumanie, en Hongrie, en Lettonie, sensiblement moindre en Slovénie, Lituanie, Bulgarie, Pologne et Estonie, et marginale en Slovaquie et en République tchèque. Par leur genèse, leurs modes de fonctionnement et leurs perspectives d'évolution, les formes sociales de production postcollectivistes demeurent singulières.

\section{Une diversité de trajectoires agraires et de profils structurels}

\section{Les limites d'une démarche classificatrice}

Pour identifier les caractéristiques des structures d'exploitation des nouveaux États membres* (NEM) et en quoi elles se distinguent de celles des anciens pays membres (les quinze États de l'Union européenne ou UE-15*), on dispose des données statistiques recueillies par Eurostat* selon des méthodologies communes à l'ensemble des États membres de l'UE. Ces catégories statistiques permettent une recension des unités de production agricole selon des critères identiques : la superficie agricole utilisée (SAU), le nombre d'unités de travail annuel (UTA), la dimension économique exprimée en production standard (PS), le statut de l'exploitation, individuelle ou personne morale. Les critères retenus par Farm Structure Survey (FSS) donnent accès à une classification des exploitations agricoles indicative de la singularité structurelle de ces agricultures. L'entrée par le niveau de concentration foncière qui privilégie le recours aux critères 
se rapportant à la taille (SAU) souligne l'existence d'un dualisme agraire marqué par la présence de grandes exploitations et d'un très grand nombre de petites unités de production de moins de 10 ha, avec une forte proportion de microexploitations de moins de 2 ha (plus de $70 \%$ du nombre total des exploitations dans les cas de la Hongrie et de la Roumanie). Si l'on répartit la SAU en huit classes selon la taille des unités de production, on constate que dans trois pays, la Slovaquie, la République tchèque, la Bulgarie, plus des quatre cinquièmes de la SAU sont occupés par des exploitations dont la surface est supérieure à 100 ha. L'importance relative de cette catégorie de taille est largement majoritaire en Hongrie, en Estonie, Lettonie, proche de la moitié de la SAU totale en Roumanie et en Lituanie, alors qu'elle est particulièrement faible en Pologne et en Slovénie dont les agricultures paysannes avaient échappé à une collectivisation* généralisée. Ce premier indicateur statistique ne règle pas la question du seuil de définition de la grande exploitation. Ce seuil doit être apprécié de manière relative dans le contexte de la diversité structurelle propre à chaque pays. En effet, c'est la présence et les traits spécifiques des très grandes exploitations, étendues sur un millier d'hectares et plus, qui distingue les structures agraires d'un certain nombre de NEM.

Figure 4. Distribution des exploitations agricoles selon les classes de dimension économique (standard output en euros, 2010)

\begin{tabular}{cccccc} 
Pays & 0 & 0 à $4000 €$ & $\begin{array}{c}4000 \text { à } \\
<25000 €\end{array}$ & $\begin{array}{c}25000 \text { à } \\
<250000 €\end{array}$ & $\begin{array}{c}250000 € \\
\text { et plus }\end{array}$ \\
$\begin{array}{c}\text { Bulgarie } \\
\text { République } \\
\text { tchèque }\end{array}$ & 0,3 & 84,6 & 12,1 & 2,5 & 0,5 \\
Estonie & 0,6 & 16,7 & 43,7 & 28,4 & 10,6 \\
Lettonie & 18,0 & 40,9 & 28,1 & 11,0 & 2,0 \\
Lituanie & 1,6 & 64,5 & 21,2 & 4,7 & 0,4 \\
Hongrie & 3,5 & 71,7 & 22,5 & 3,9 & 0,3 \\
Pologne & 2,8 & 48,0 & 14,7 & 3,5 & 0,4 \\
Roumanie & 2,6 & 88,7 & 38,6 & 9,5 & 0,4 \\
Slovénie & 0 & 46,0 & 10,7 & 0,6 & 0,1 \\
Slovaquie & 1,3 & 44,7 & 45,2 & 9,9 & 0,2 \\
\hline
\end{tabular}

Source : Eurostat*, 2013 (online data code : ef_kvecsleg)

Le nombre considérable de petites exploitations constitue la deuxième spécificité des structures agraires. Encore faut-il s'entendre sur ce que l'on range dans cette catégorie, en l'absence de définition commune à l'échelle européenne. Les critères ont trait à la superficie agricole utile, au volume de travail, au niveau de l'autoconsommation, à la taille économique. Le seuil de la petite exploitation peut être défini en termes absolus ou relatifs (en fonction de la distribution statistique propre à chaque pays). On range dans cette catégorie les exploitations d'une taille inférieure à 5 ha. En Bulgarie, Hongrie, Roumanie, les quatre cinquièmes et plus des exploitations recensées ont moins de 5 ha. En Slovaquie, Slovénie, Lituanie la part relative de cette catégorie dépasse la moitié du 
nombre total des exploitations. Prises ensemble, ces petites exploitations de moins de 5 ha n'occupent qu'une faible part des terres, généralement inférieure à un dixième de la SAU, à l'exception de la Roumanie où elles forment plus des neuf dixièmes du nombre total et couvrent $28,4 \%$ de la SAU. La comparaison est cependant biaisée du fait qu'en 2010, la République tchèque, la Slovaquie et la Pologne ont relevé le seuil respectivement à 5 ha, 2 ha et 1 ha, ce qui réduit la couverture de cette catégorie d'exploitations suivies par FSS.

Cette dualité structurelle s'exprime plus fortement encore en termes de dimension économique (Eurostat*, 2014). Les données d'Eurostat soulignent que plus des quatre cinquièmes des unités fournissent moins de 4000 euros de production standard (PS) en Roumanie et en Bulgarie, et autour de la moitié encore dans les autres pays, à l'exception de la République tchèque. Dans ce dernier pays, ainsi qu'en Slovaquie, les trois quarts de la SAU sont mis en valeur par de grandes exploitations agricoles (plus de 250000 euros).

Figure 5. Répartition de la SAU selon l'importance du travail familial au sein de l'exploitation (en \%)

\begin{tabular}{|c|c|c|c|c|}
\hline Pays & $\begin{array}{l}\text { Travail familial } \\
\text { exclusif }\end{array}$ & $\begin{array}{l}\text { Travail familial } \\
<50 \%\end{array}$ & $\begin{array}{l}\text { Travail familial } \\
\quad>50 \%\end{array}$ & $\begin{array}{l}\text { Sans travail } \\
\text { familial }\end{array}$ \\
\hline Bulgarie & 23,8 & 4,8 & 10,9 & 60,6 \\
\hline $\begin{array}{l}\text { République } \\
\text { tchèque }\end{array}$ & 16,8 & 5,6 & 6,6 & 71,0 \\
\hline Estonie & 37,5 & 6,7 & 3,6 & 52,2 \\
\hline Lettonie & 62,4 & 13,8 & 22,7 & 1,1 \\
\hline Lituanie & 67,8 & 9,0 & 9,7 & 13,5 \\
\hline Hongrie & 44,7 & 4,7 & 2,2 & 48,3 \\
\hline Pologne & 83,2 & 3,0 & 2,9 & 10,9 \\
\hline Roumanie & 56,0 & & & 44,0 \\
\hline Slovénie & 93,6 & 0,6 & 0,3 & 5,5 \\
\hline Slovaquie & 13,3 & 2,9 & 3,1 & 80,7 \\
\hline
\end{tabular}

Source : Eurostat*, 2013.

D’autres modes de catégorisation peuvent être croisés avec une approche par taille. Les données publiées par Eurostat* classent les exploitations en fonction de l'apport en travail de la main-d'œuvre familiale (selon trois niveaux, exclusivement familial, plus de $50 \%$ ou moins de $50 \%$ de l'apport total). L'absence de travail familial devient alors le critère discriminant définissant par défaut une catégorie en marge de l'agriculture dite familiale. La définition de l'agriculture familiale* renvoie à un mode d'organisation de l'activité productive associant famille et exploitation. Cette catégorie normative englobe un très large éventail de tailles et de situations, de la petite exploitation de semisubsistance jusqu'à la grande exploitation relevant d'un mode de gestion patronale 2 .

2. Un rapport européen analyse la grande diversité de l'agriculture familiale, à laquelle les NEM ne font pas exception (Davidova et Thomson, 2014). 
La main-d'œuvre familiale apporte une contribution prépondérante en Pologne et en Slovénie (plus des neuf dixièmes du montant des « unités de travail annuel*»UTA), en Roumanie (plus des quatre cinquièmes), en Lettonie et en Lituanie (plus des trois quarts), et une part majoritaire en Hongrie et en Estonie. Bien qu'en petit nombre, les exploitations ayant la personnalité morale peuvent occuper une place significative pour ce qui est de la SAU en Estonie (52,2 \%), en Hongrie (48,3 \%) et en Roumanie (44 \%). Cette forme d'agriculture faisant appel au travail salarié (plus des quatre cinquièmes des UTA*) est largement dominante en Slovaquie $(80,7 \%$ de la SAU), en République tchèque $(71 \%)$ et en Bulgarie (60,6\%).

Si les catégories descriptives et normatives soulignent les singularités structurelles de ces agricultures, elles présentent certaines limites.

\section{Des profils structurels différenciés}

À des degrés divers, ces agricultures portent l'empreinte d'héritages structurels de longue portée propres à l'Europe des grands domaines où les réformes agraires, tardivement engagées dans la première moitié du $\mathrm{xx}^{\mathrm{e}}$ siècle, sont restées inachevées, privant les paysanneries d'un accès stable à la propriété de leurs terres. La collectivisation*, puis la décollectivisation* ont déclenché de nouvelles mutations des structures foncières sans effacer complètement les héritages agraires mais en les remaniant pour composer des trajectoires dépendantes des choix du passé et des itinéraires de sortie du collectivisme. Des similitudes de profils permettent de distinguer quelques grands types de trajectoires d'évolution.

Figure 6. Nombre d'exploitations par catégories de taille en 2013

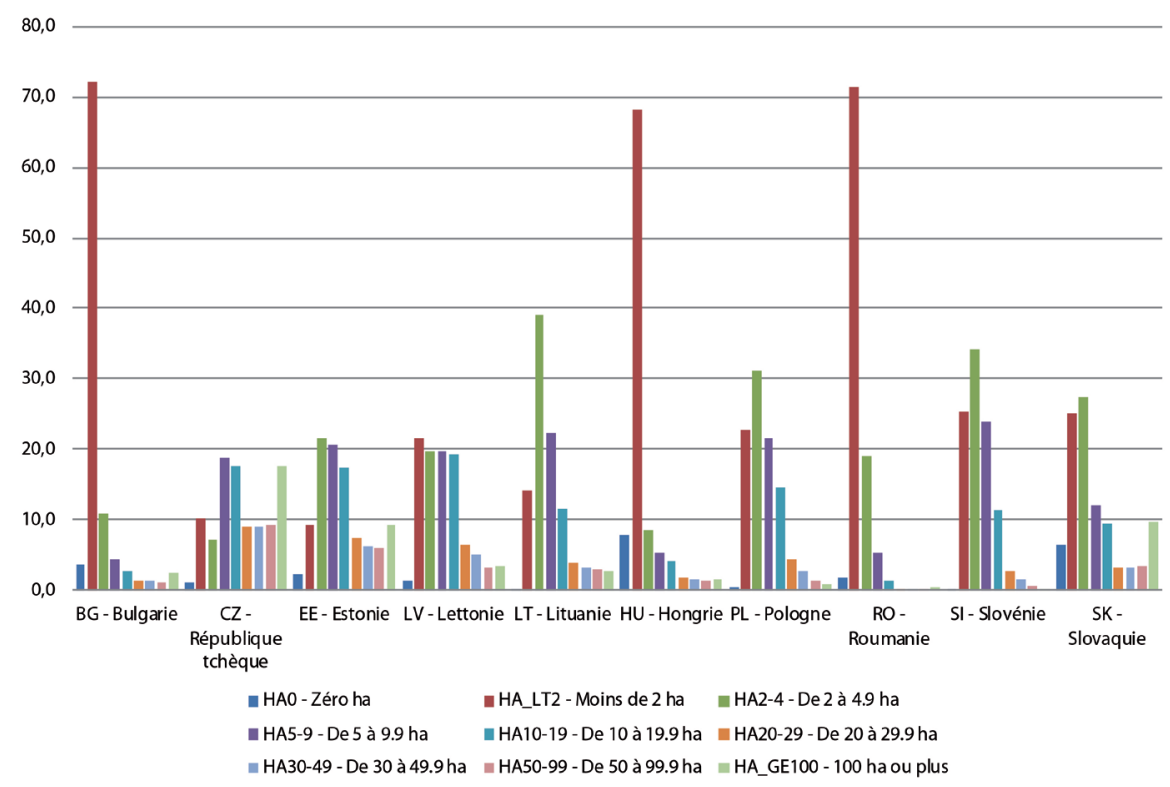


Figure 7. Répartition de la SAU en \% selon les catégories de taille en 2013

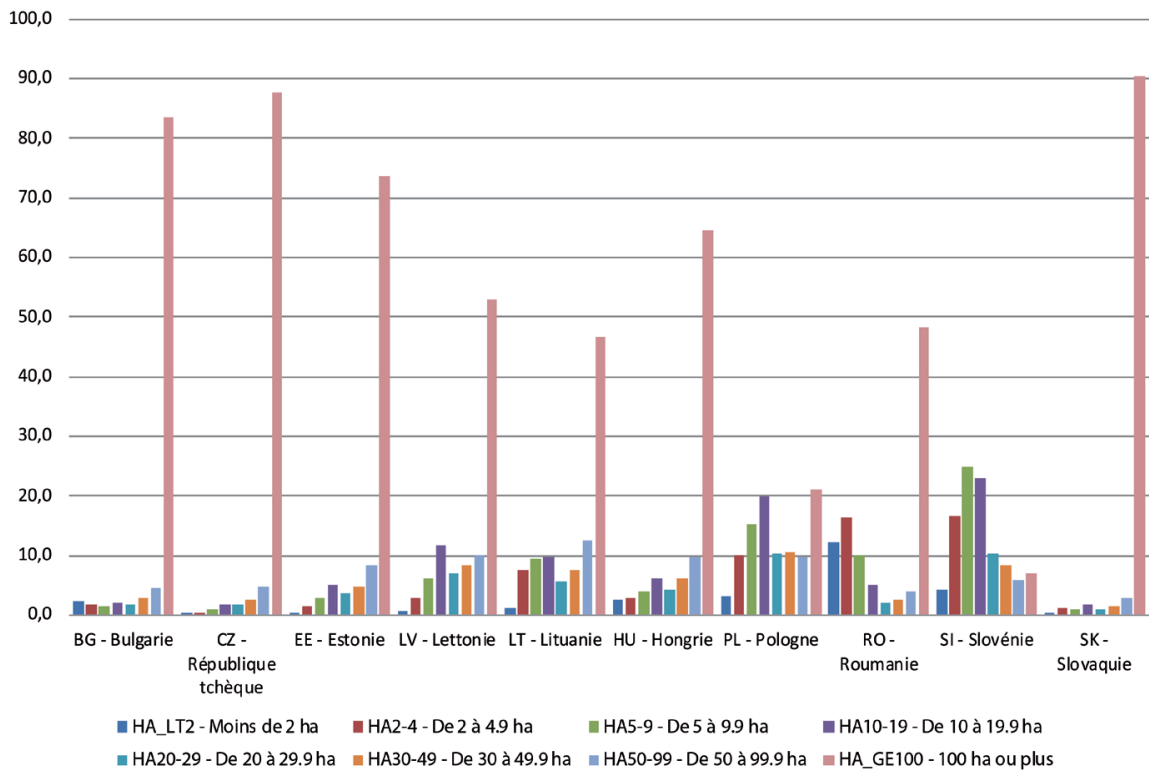

\section{Des agricultures à dominante de grandes exploitations sociétaires}

Elles se caractérisent par un degré élevé de concentration foncière, un mode de faire-valoir indirect largement majoritaire, un salariat dominant. Le niveau de l'emploi agricole, après un fort délestage imposé par les restructurations, y est en contraction continue. Cette situation concerne en premier lieu la République tchèque, la Slovaquie, l'Estonie et la Bulgarie dont les agricultures répondent aux critères précédemment définis.

En République tchèque comme en Slovaquie, la transformation postcollectiviste a été fondée sur la restitution des biens confisqués par le pouvoir communiste (après février 1948) et sur la restructuration des coopératives et la privatisation des fermes d'État. Née de l'application d'un dispositif législatif complexe, la restructuration a suscité des conflits d'intérêts entre diverses catégories d'acteurs sociaux impliqués dans le processus de transformation, notamment au sujet du contrôle du capital des exploitations successeurs ${ }^{3}$.

L'agriculture tchèque a connu une reconstitution limitée du modèle de l'exploitation familiale. Au début de la décennie 1990, la restitution de terres aux anciens propriétaires aurait pu favoriser l'installation d'exploitants indépendants. Au départ, celle-ci a été encouragée par une politique de soutien à l'investissement, mais, à partir de 1994, le mouvement de réappropriation au profit d'exploitants individuels s'est ralenti. La couche sociale susceptible de porter un projet d'exploitation familiale marchande était relativement étroite. Les travailleurs des exploitations collectives (discriminés par les critères d'attribution des parts de capital) n'étaient guère intéressés. Les nouveaux

3. C'est-à-dire les anciennes exploitations collectives après conversion de leur statut juridique et attribution des parts de capital entre les ayants droit. 
exploitants se sont recrutés parmi les anciens cadres dirigeants et le groupe des spécialistes (ingénieurs, techniciens). Dans la deuxième moitié des années 1990, les coopératives de propriétaires ont traversé une deuxième vague de transformation, marquée par une appropriation-contrôle de leur capital d'exploitation au profit des " managers » et une conversion de leur statut juridique en formes sociétaires*. Par ailleurs, la privatisation des anciennes fermes d'État a donné majoritairement naissance à des entreprises en société, plus rarement à des exploitations en responsabilité personnelle. Les terres et les biens (bâtiments, équipements) ont été mis en location par le fonds foncier d'État avant que ne soit engagée leur mise en vente.

En dépit d'une fragmentation de la propriété foncière, l'usage des terres est resté concentré au bénéfice de grandes structures de production qui louent les terres. Seulement $16 \%$ de la superficie agricole du pays est en faire-valoir direct. Près des neuf dixièmes de la SAU sont cultivés par de grandes exploitations de plus de 100 ha. Au nombre de 4630 unités de production, en 2013, elles forment 17,6\% du nombre total, et cultivent des surfaces importantes (en moyenne 662 ha de SAU), principalement en faire-valoir indirect (88,5\%). Pour moitié, ce sont de grandes exploitations en responsabilité personnelle, pour une autre moitié des entreprises sociétaires. Leur charge en main-d'œuvre reste élevée bien que le processus de substitution du travail par le capital s'accélère puisqu'on observe une réduction du volume de la force de travail d'environ un tiers pour la période 2005-2013. Prises ensemble, ces grandes exploitations réalisent $78 \%$ en valeur de la production standard. Ces grandes unités se sont ajustées aux nouvelles relations de prix imposées par l'ouverture du marché, en se spécialisant dans la production végétale (céréales, oléagineux, cultures industrielles) et en cherchant à améliorer la productivité du travail.

À ce premier type, on peut rattacher le profil de l'agriculture bulgare qui se caractérise par un haut niveau de concentration foncière au profit de la grande exploitation. La liquidation des anciennes coopératives de production s'est opérée dans le contexte d'une transition bousculée par d'incessants retournements de majorité politique qui ont entrainé à deux reprises la révision de la loi foncière adoptée en 1991. La restitution des terres, sur la base des anciens cadastres datant d'avant 1946, a créé une masse de petits propriétaires virtuels et suscité de nombreux contentieux. Des dispositions, introduites en 1995, prévoyaient de remembrer les parcelles dans l'objectif de favoriser leur transfert aux nouvelles coopératives de propriétaires tandis que les exploitants individuels se voyaient attribuer des parcelles éloignées ou de moindre qualité. La reprivatisation a provoqué l'émiettement de la propriété foncière entre les mains d'une multitude d'ayants droit qui, dans leur grande majorité, n'envisageaient pas de les exploiter et qui les ont données à bail aux coopératives d'une part, et aux entreprises constituées par les arendatori, un groupe formé par les anciens cadres dirigeants, d’autre part. De manière générale, la désorganisation du secteur agricole s'est traduite par l'effondrement de la production, l'abandon d'une partie des surfaces cultivées, une dépopulation accélérée des villages. Cette période de trouble a été mise à profit par l'ancienne élite agraire pour faire main basse sur le capital d'exploitation et engager un processus de concentration des terres dans le cadre de contrats de location à court terme. À partir des années 2000, l'emprise foncière de ces grandes exploitations de type entrepreneurial* s'affirme et se consolide. Entre 2003 et 2010, le nombre des exploitations diminue de plus de la moitié $(-52,4 \%)$. Les exploitations de plus de 50 ha accroissent leur importance relative, passant de $78 \%$ à $87 \%$ de la SAU. Ce processus d'accumulation foncière s'accomplit par le biais de la 
location auprès de nombreux petits propriétaires, en majorité citadins (les deux tiers de la SAU sont en faire-valoir indirect). En marge de ce noyau de grandes exploitations cultivant majoritairement des céréales et des protéagineux, une masse d'exploitations microfundiaires de moins de 5 ha, représentant 85,3\% du nombre total des exploitations recensées, se partagent $4 \%$ de la SAU, dévolus à la production de fruits et légumes, et à l'entretien d'un petit cheptel fournissant lait et viande.

\section{Des agricultures marquées par la dualité structurelle}

La dualité des structures agraires est la trace d'un affrontement de longue durée entre la grande et la petite exploitation, sous la forme d'un partage inégal entre les grands domaines et les tenures* paysannes, puis entre les exploitations collectives et les lopins de l'économie auxiliaire. Ce caractère dual a été refaçonné par l'abolition de l'appropriation collective sur la terre et le retour à la petite propriété individuelle, sur la base d'une trame foncière remontant aux réformes agraires précédant la collectivisation*. Le nombre pléthorique de toutes petites exploitations et la présence de grandes exploitations mettant en valeur une part prépondérante des terres constituent les traits propres de ce type de profil. Les formes prises par la décollectivisation et leur inégal encadrement par des dispositifs juridiques opposent une transition hongroise, en apparence ordonnée, au démantèlement brutal et chaotique des structures collectives en Roumanie.

En Hongrie, la réaffectation des droits de propriété a donné naissance à une relative diversité de formes sociales de production. Le retour des terres aux anciens propriétaires a suivi la voie originale des compensations (sous forme de bons d'une valeur correspondant à celle du bien confisqué). Le dispositif prévoyait que les anciennes exploitations collectives devaient affecter une partie des terres pour les mettre en vente aux enchères auprès des porteurs de bons de compensation. Par ailleurs, une portion importante des terres, restée propriété des membres des coopératives ont pu être retirées des structures collectives par leurs propriétaires. Enfin, des parcelles de terre ont été attribuées aux employés des exploitations collectives qui n'en possédaient pas. La transition postcollectiviste s'est soldée par un ample mouvement de fragmentation de la propriété foncière : un demi-million de personnes ont reçu plus de deux millions d'hectares en moins de cinq ans. Dispersés entre un grand nombre de petits propriétaires parcellaires, les droits de propriété ont été dissociés des droits d'usage détenus par des structures de grande taille et de statut divers, cultivant les terres dans le cadre de baux de location. Si la loi sur la terre, adoptée en 1994, interdit l'achat de terres aux coopératives et aux formes sociétaires*, en revanche elle autorise leur location auprès des propriétaires fonciers ${ }^{4}$. Les deux tiers de la SAU sont en faire-valoir indirect.

Si la trajectoire hongroise porte la marque d'une dualité structurelle initiale, elle évolue progressivement vers une plus grande diversité de structures quant à la taille, au statut de la main-d'œuvre, à l'orientation de l'activité productive. Transformées en coopératives de propriétaires, les anciennes exploitations collectives ont d'abord entrepris de s'ajuster aux nouvelles règles de l'économie de marché mais, à partir de 2000, le processus de recomposition du capital s'est accéléré, favorisant la conversion des coopératives en macroexploitations sociétaires. Le nombre des exploitations individuelles a diminué d'un tiers de 2005 à 2013, passant de 706900 unités à 483 520, cependant que

4. Les personnes privées ne peuvent louer plus de 300 ha alors que le plafond pour les entités légales (coopératives et sociétés) est fixé à 2500 ha. 
leur taille moyenne augmentait de 3 à 5,1 ha. La grande majorité de ces petites unités de production relèvent d'une agriculture de subsistance. Représentant 84,6 \% du nombre total, les exploitations de moins de 5 ha cultivent $5,4 \%$ de la SAU, et leur production est en majorité destinée à la consommation familiale. La présence d'exploitations en responsabilité personnelle dont la taille est comprise entre 5 ha et moins de 100 ha, qui forment $13,8 \%$ du nombre et couvrent 32,1\% de la SAU, est un trait propre à une trajectoire de privatisation qui a permis l'accès au capital foncier d'une couche d'exploitants dotés d'un capital technique et d'un esprit d'entreprise. La catégorie des grandes exploitations de plus de 100 ha est elle-même composite, formée pour plus de la moitié d'exploitations en responsabilité personnelle de taille limitée (191 ha en moyenne) et d'entreprises sociétaires* dont la superficie moyenne est plus de trois fois supérieure (725 ha). Le profil de l'agriculture hongroise apparait relativement diversifié tant du point de vue des statuts juridiques, des catégories de taille que des formes de travail (familiale ou salariée).

Le profil de l'agriculture roumaine est marqué par une dualité structurelle extrême. Expression d'une filiation agraire de longue durée et produit du démantèlement brutal des cadres collectifs imposés au monde paysan, le réaménagement de cette dualité est source de difficultés. Plus de deux décennies après la fin du collectivisme, la charge agraire de l'espace rural, matérialisée par une masse de microstructures, reste très forte. Ces petites unités coexistent avec la présence de très grandes exploitations dont certaines atteignent plusieurs milliers d'hectares et comptent parmi les plus étendues en Europe. En révélant des tensions sociales anciennes et puissantes, la rupture avec le collectivisme a fait ressurgir une question agraire lancinante, jamais définitivement réglée. Mise en œuvre dans l'urgence, par l'adoption de la loi sur le foncier*, la redistribution de 9 millions d'hectares à 5,6 millions de propriétaires a émietté la trame agraire en faisant bondir le nombre des exploitations de moins de cinq hectares et le plus souvent de moins de deux ${ }^{5}$. Une logique distributive animée par un idéal paysan égalitaire a prévalu sur un objectif de viabilité économique d'unités de production qui se sont trouvées démunies des équipements et de l'encadrement technique et financier indispensables. La « loi sur les sociétés agricoles* et autres formes d'association en agriculture » (loi 36/1991) prévoyait la recomposition de l'appareil productif autour d'associations agricoles inspirées de principes coopératifs. Par ailleurs, le souci de ne pas démembrer les anciennes fermes d'État qui bénéficiaient d'un bon niveau d'équipement technique, a conduit à les restructurer juridiquement sous la forme de sociétés commerciales à participation publique, donnant ainsi naissance à un noyau de grandes structures productives dont la privatisation effective n'a pas été engagée avant l'an 2000.

La distinction entre deux grandes catégories, les entreprises commerciales, dotées de la personnalité juridique, et les exploitations agricoles individuelles (qui peuvent avoir un statut de personne physique autorisée), recouvre schématiquement le partage entre un petit groupe d'exploitations de plus de 100 ha cultivant $48 \%$ de la SAU (2013) et une pléthore d'exploitations de moins de 5 ha, comptant plus de 3,5 millions d'unités et détenant $28,5 \%$ de la SAU. Entre ces deux pôles, un continuum d'exploitations petites et moyennes $(7,4 \%$ du nombre total $)$ met en valeur moins du quart de la SAU. Le rapport

5. La loi 18/1991 sur le fonds foncier a rétabli la libre disposition des biens fonciers et a posé le principe de la reconstitution de la propriété privée sur les terres, à l'exclusion des terres affectées au domaine public. Elle a autorisé la dissolution des coopératives et la redistribution des terres à leurs anciens propriétaires (avec un plafond de $10 \mathrm{ha}$ ) et l'attribution de parcelles d'un minimum de 0,5 ha à tous les membres. La restitution rétablit une trame foncière émiettée. 
entre les microexploitations paysannes et les grandes entreprises s'articule différemment selon les régions. Les plaines de grande culture, dominées par les parcellaires à large maille des exploitations sociétaires* (sociétés commerciales) s'opposent aux zones de collines et de montagnes des Carpates où le poids du microfundium paysan l'emporte. Les grandes entreprises commerciales ne cessent de renforcer leur emprise foncière, avec le soutien des investisseurs agro-industriels* domestiques et étrangers, tandis que les petites exploitations de polyculture, tournées vers l'autoconsommation, jouent le rôle de filet social de sécurité assurant la subsistance des groupes les plus vulnérables (chômeurs, retraités, personnes au foyer).

\section{Des agricultures familiales à dominante de petites et moyennes exploitations}

La conversion structurelle de l'agriculture lituanienne procède d'une trajectoire qui, renouant avec l'héritage de la réforme agraire* des années 1920 (lors de la première indépendance), a conduit à réhabiliter l'exploitation familiale. Bien que marqué du sceau de l'alternance politique, le processus de réforme agraire* a pris appui sur le principe de restitution des terres à leurs anciens propriétaires ${ }^{6}$, tout en préservant le droit d'usage sur les lopins attribués aux ménages ruraux. La méthode de privatisation des biens collectifs a permis que se constituent de petites entreprises ainsi que des associations de producteurs agricoles (à l'initiative des cadres dirigeants et des membres des anciens collectifs). De plus petite taille que les exploitations collectives, ces associations ne se sont pas révélés viables si bien que leur place n'a cessé de décroître. Dès le moment initial de la privatisation, une partie des ayants droit a saisi l'opportunité de retirer leurs terres des structures collectives, en emportant une part du capital qui leur revenait (généralement en nature sous forme de machines, cheptel ou équipements). Ces stratégies actives de réappropriation ont été le fait de groupes de taille limitée, souvent familiaux. Les plus entreprenants sont parvenus par des achats ou des locations à se doter d'un outil foncier plus étendu que celui possédé par leur famille autrefois et à asseoir ainsi une exploitation de taille viable.

Le recensement agricole de 2010 distingue deux catégories d'exploitations : d'une part, les exploitations familiales et en responsabilité personnelle, largement majoritaires (au nombre de 199 267) occupant $87 \%$ de la SAU et fournissant $77 \%$ de la production brute standard, et d'autre part, les entreprises et sociétés agricoles* en plus petit nombre (646), avec une taille moyenne de 573 ha et cultivant $13 \%$ de la SAU. Plus de la moitié $(53,3 \%)$ des exploitations ont moins de 5 ha et occupent à peine $8,8 \%$ de la SAU. Le noyau de ces microexploitations est formé par les bénéficiaires des trois hectares attribués aux ménages ruraux. Mobilisant le travail des membres de la famille, elles sont orientées vers l'autoconsommation et pratiquement exclues du marché.

Le choix politique opéré en faveur de la restauration de la propriété privée de la terre a permis que se régénère un authentique modèle d'agriculture familiale* qui renoue avec l'expérience vécue pendant la période d'indépendance de l'entre-deux-guerres. Les tendances de l'évolution révèlent que les exploitations familiales de type marchand, au-delà du seuil de 30 ha, se consolident, portées par un élargissement de leur assise foncière et une spécialisation orientée vers les produits de l'élevage.

6. Principe assorti de dispositions limitant les superficies restituables. Les seuils ont été modifiés à plusieurs reprises (plafonds de 80 ha dont 50 ha de terres agricoles, par la suite fixés à 150 ha). 
Au sein du bloc de l'Est, la Pologne et la Slovénie faisaient figure d'exception en 1989. On ne retiendra ici que le cas de l'agriculture polonaise. La Pologne a pu faire l'économie d'une décollectivisation* de grande ampleur, toutefois des changements sont intervenus en termes de propriété et d'usage des terres, et de modalités d'insertion des exploitations individuelles dans l'économie de marché (Halamska, 2011). Le secteur étatique, et plus encore le secteur coopératif, y étaient d'importance minoritaire par rapport à un secteur privé, composé de petites exploitations individuelles détenant 76,2\% de la superficie agricole. Dans les années 1992-1995, les fermes d’État ont été liquidées et leurs terres transférées à une agence de la propriété agricole du Trésor public, en charge de la gestion de ce patrimoine foncier. L'agence a privilégié les exploitations sociétaires* pour l'attribution des terres en location ou pour leur mise en vente. La restructuration des biens de l'ancien secteur socialisé (fermes d'État et coopératives de production agricole) s'est soldée au bénéfice de l'élargissement des exploitations familiales et de l'émergence d'une agriculture sociétaire (de statut privé), en majorité formée d'un petit nombre de grandes exploitations et de coopératives de production qui ne contrôlent plus que $9,1 \%$ de la SAU.

Les dynamiques internes à l'agriculture familiale* révèlent un processus de différenciation relatif aux conditions et aux possibilités d'intégration des diverses catégories dans l'économie de marché. Une première tendance concerne la réduction progressive du nombre d'exploitations qui prolonge et amplifie une tendance observée depuis les années cinquante. Durant les années 1990, le rythme de diminution reste relativement lent, les exploitations se retirent des circuits marchands mais ne disparaissent pas, les petites exploitations de moins de 5 ha enregistrent le plus fort recul tandis qu'augmente le nombre des exploitations de plus de 5 ha et surtout de plus de 20 ha. Au lendemain de l'entrée dans l'UE, le rythme de disparition du nombre des unités de production s'intensifie ( $-42,3 \%$ entre 2005 et 2013). La réduction affecte les plus petites exploitations (moins de $5 \mathrm{ha}$ ) tandis que progresse le nombre des exploitations supérieures à 20 ha.

En 2013, on dénombre 1429010 exploitations dont plus de la moitié (54,4\%) ont une taille inférieure à cinq hectares et détiennent $13 \%$ de la SAU tandis que les exploitations de 20 ha et plus, représentant moins du dixième du nombre total, détiennent plus de la moitié de la SAU. Si l'on retient le critère de la dimension économique, la moitié des exploitations réalisent moins de 4000 euros de production standard, la plupart ne produisant que pour leurs propres besoins. L'agriculture familiale polonaise est désormais constituée d'unités de production ayant des caractéristiques et des logiques de fonctionnement différentes (Maurel et al., 2003). Deux trajectoires d'évolution coexistent : l'une, ascendante, se caractérise par une plus grande intégration au marché d'exploitations de bonne taille qui élargissent leur assise et deviennent plus intensives, l'autre descendante, procède d'un retrait progressif du marché qui s'accompagne d'un repli de la production (simplification du système de cultures, abandon des élevages). Devenue la forme sociale prépondérante, l'agriculture familiale est traversée par une nouvelle forme de dualité structurelle :

1. Une agriculture marchande fonctionnant dans un modèle de type « entreprise familiale », engagée dans des stratégies d'accumulation foncière, constitue le pôle moderniste. Dans leur grande majorité, ces exploitants cherchent à élargir leur assise foncière mais n’y parviennent que difficilement. La réalisation de ce modèle familial marchand est limitée par la survie, largement assistée, d'une majorité de petites exploitations de subsistance jouant le rôle de « filet de sûreté ». 
2. Une agriculture de subsistance, en voie de marginalisation sur le plan économique, forme le gros des bataillons de l'agriculture familiale sur de petites surfaces, aux mains d'agriculteurs souvent âgés. Retirés du marché, ces chefs d'exploitation se vivent comme des « quasi-paysans » mais en réalité tirent l'essentiel de leurs revenus d'autres sources (notamment des revenus sociaux) que de l'activité agricole. Si ces exploitations ont commencé à réduire leur assise foncière, en louant, voire en vendant quelques parcelles, une autre partie conserve son patrimoine foncier, bloquant ainsi les possibilités d'agrandissement des autres exploitations. L'insuffisante fluidité du marché foncier constitue une entrave à la restructuration.

\section{Scenari de concentration foncière et de restructuration}

Les structures agraires, issues du processus de décollectivisation*, poursuivent leur transformation sur un rythme soutenu. Depuis l'entrée dans l'UE, le nombre d'exploitations enregistre une forte diminution tandis que la SAU reste stable ou s'accrôt avec la remise en culture des terres qui avaient été un temps laissées en friche. Les tendances à la concentration au profit des grandes exploitations de type sociétaire* se renforcent.

Figure 8. Évolution du nombre total des exploitations et de la SAU (2005-2013)

\begin{tabular}{|c|c|c|c|c|c|c|}
\hline Pays & $\begin{array}{c}\text { Nombre } \\
\text { d'exploi- } \\
\text { tations } \\
2005\end{array}$ & $\begin{array}{c}\text { Nombre } \\
\text { d'exploi- } \\
\text { tations } \\
2013\end{array}$ & $\begin{array}{c}\text { Variation } \\
2005- \\
2013 \\
\text { (en \%) }\end{array}$ & $\begin{array}{c}\text { SAU en } \\
2005\end{array}$ & $\begin{array}{c}\text { SAU en } \\
2013\end{array}$ & $\begin{array}{c}\text { Variation } \\
2005- \\
2013 \\
\text { (en \%) }\end{array}$ \\
\hline Bulgarie $^{7}$ & 534610 & 254410 & $-52,4$ & 2729390 & 4650940 & 70,4 \\
\hline $\begin{array}{l}\text { République } \\
\text { tchèque }\end{array}$ & 42250 & 26250 & $-37,9$ & 3557790 & 3491470 & $-1,9$ \\
\hline Estonie & 27750 & 19190 & $-30,8$ & 828930 & 957510 & 15,5 \\
\hline Lettonie & 128670 & 81800 & $-36,4$ & 1701680 & 1877720 & 10,3 \\
\hline Lituanie & 252950 & 171800 & $-32,1$ & 2792040 & 2861250 & 2,5 \\
\hline Hongrie & 714790 & 491330 & $-31,3$ & 4226550 & 4656520 & 9,1 \\
\hline Pologne & 2476470 & 1429010 & $-42,3$ & 14754880 & 14409870 & $-2,3$ \\
\hline Roumanie & 4256150 & 3629660 & $-14,7$ & 13906700 & 13055850 & $-6,1$ \\
\hline Slovénie & 77170 & 72380 & $-6,2$ & 485430 & 485760 & 0,1 \\
\hline Slovaquie & 68490 & 23570 & $-65,6$ & 1879490 & 1901610 & 1,2 \\
\hline
\end{tabular}

Source : D’après Eurostat*, 2013 ; calculs de l'auteur.

7. Dans le cas de la Bulgarie, l'accroissement de la SAU entre les deux dates, résulte en partie de l'inclusion des terres communes dans la SAU, à partir du recensement de 2010, ce qui compromet la comparaison entre les données. 
Les petits exploitants individuels ont subi les effets déstabilisateurs de la privatisation du système agroalimentaire et des circuits de distribution, puis de la pénétration sur le marché domestique des produits venus d'Europe occidentale.

Les États d'Europe centrale ont rencontré des obstacles pour construire leur propre référentiel de politique agricole. Dans un contexte de relative instabilité des scènes gouvernementales, des changements de priorités concernant le modèle d'agriculture à soutenir ont pu se produire ${ }^{8}$.

\section{Les effets de la PAC sur les transformations agraires : un ciblage inapproprié}

L'entrée des nouveaux États membres dans l'Union (en 2004 ou en 2007) et la mise en œuvre du dispositif de la PAC ont eu un impact manifeste sur les évolutions structurelles. L'introduction progressive de mesures de soutien destinées à appuyer la restructuration dans une perspective de convergence avec les anciens États membres, s'est opérée sans que l'on s'interroge sur leur adéquation au vu des contextes propres à ces agricultures. Or, le référentiel politique du « modèle opérationnel dominant en Europe », c'est-à-dire de l'agriculture familiale*, s'est révélé inapproprié.

Mises en application dans le cadre du régime simplifié de paiements directs, les mesures de soutien du premier pilier* ont favorisé les grandes exploitations agricoles. En dépit du relatif élargissement du nombre de bénéficiaires", l'aide directe versée aux petits exploitants, compte tenu des faibles taux de paiement à l'hectare, ne peut constituer une solution pour ces petites structures. Les nombreux bénéficiaires ne perçoivent qu'une très faible part des paiements directs et leur versement est assujetti au respect d'exigences minimales.

Les mesures du second pilier de la PAC* inscrites dans la politique de développement rural adoptée par ces pays ne sont guère plus favorables aux petites structures ${ }^{10}$. À la condition qu'elles s'engagent davantage dans les circuits marchands, les exploitations dites de « semi-subsistance » peuvent bénéficier d'un dispositif d'aide à la restructuration (la mesure 141), destiné à les rendre économiquement viables. D’autres mesures proposées dans le cadre du second pilier de la PAC* viennent soutenir la modernisation des exploitations, mais les conditions d'accès, à partir d'un seuil d'éligibilité variable selon les pays, découragent les exploitants susceptibles d'en bénéficier. D’une manière générale, si les aides du premier pilier sont largement sollicitées par les exploitants, celles du second pilier semblent manquer leur cible. C'est ainsi qu'en Roumanie, « le ciblage des aides sur les exploitations de plus de 1 ha et sur les parcelles de plus de 0,3 ha vise

8. Dans certains pays, les politiques nationales ont soutenu la constitution d'exploitations de type familial, dans d'autres, elles ont privilégié l'appui aux grandes exploitations sociétaires, proches de l'agrobusiness. Ces choix ressortissent de l'inégale capacité d'intervention des organisations sociales et/ou professionnelles représentant les intérêts des diverses catégories.

9. Les ajustements opérés en faveur des plus petites exploitations ont toutefois permis d'abaisser le seuil de la superficie éligible et d'augmenter le montant attribué.

10. Les mesures du premier pilier concernent l'aide à la modernisation des exploitations ; l'aide au départ en préretraite ; l'aide aux jeunes agriculteurs. Celles relevant du second pilier, dites de « soutien à la durabilité des zones rurales » ont trait d'une part, à la diversification des activités et d'autre part, aux aides agroenvironnementales. Les premières s'appliquent à des exploitations cherchant à s'agrandir en développant le capital productif, les secondes s'adressent à des exploitations s'orientant vers des trajectoires de diversification. 
à limiter le saupoudrage d'aides aux montants dérisoires, distribuées à une multitude de microexploitations. Ce ciblage est aussi conforme à un schéma qui considère implicitement que les plus petites exploitations sont vouées à une disparition prochaine : aucun dispositif d'aide ne leur est donc destiné » (Darrot et al., 2011). De fait, les grandes exploitations sociétaires* sont les principales bénéficiaires du régime simplifié des paiements directs mais aussi des aides à la modernisation qui ont pour effet de renforcer la polarisation des structures ${ }^{11}$.

Les effets distributifs de la PAC jouent en faveur de la seule catégorie des grandes exploitations en marginalisant les autres formes sociales de production. Loin de contribuer au développement d'une agriculture de type familial, la PAC a eu pour résultat de conforter la position des grandes structures issues de la transformation des exploitations collectives tandis qu'elle rendait improbable la consolidation d'exploitations familiales de dimensions viables. Le ciblage des politiques de soutien par la catégorisation tel que le soutien accordé aux exploitations de semi-subsistance semble inopérant. Le référentiel du modèle agricole européen s'est révélé largement inapproprié à la réalité socioéconomique des agricultures centre-européennes.

\section{Des menaces d'accaparement foncier* et de dépossession}

La situation foncière des agricultures postcollectivistes reste paradoxale : l'extrême fragmentation de la propriété foncière ne se traduit pas dans la trame d'usage des terres caractérisée par une relative concentration au profit des grandes exploitations. Une proportion largement dominante de leurs terres est en faire-valoir indirect. Les modalités de la concentration foncière sont diverses en fonction des caractéristiques du marché de la terre. Longtemps resté peu animé, le marché foncier est à présent soumis à des évolutions rapides du fait de la fin du moratoire qui interdisait l'achat des terres par les étrangers ${ }^{12}$. Dans la plupart de ces pays, la pénétration des investisseurs européens et internationaux accélère le processus d'accaparement foncier* au bénéfice des très grandes exploitations ce qui a pour effet de marginaliser les petites et moyennes structures.

En Hongrie, de 2000 à 2010, le nombre des petites exploitations agricoles a enregistré un fort recul de l'ordre de $40 \%$, passant de 996900 à 577000 unités d'une taille moyenne de 4,6 ha. Après l'accession à l'UE, la majorité des petites exploitations s'est trouvée exclue des aides de la PAC tandis que le système de paiement unique à l'hectare accélérait la concentration des terres au profit des grandes structures. Le faible prix de la terre, comparativement aux pays d'Europe occidentale, a attiré les investisseurs étrangers qui, en dépit de l'interdiction d'achat, ont réussi à mettre la main sur des superficies étendues, dans le cadre de «contrats de poche $»^{13}$. L'attitude de l'État hongrois à l'égard de la frange d'investisseurs responsables de l'accaparement des terres* a été et reste pour le moins ambiguë. De 2002 à 2010, le transfert des terres appartenant au domaine public au profit de groupes d'investisseurs s'est développé de manière continue et le processus d'accaparement a été facilité par l'alliance du capital bancaire avec les élites politiques ${ }^{14}$.

11. En 2013, en Roumanie 1,1\% des bénéficiaires ont reçu 51,7\% des paiements directs ; en Bulgarie 45,6 \%. En Hongrie moins de $1 \%$ des bénéficiaires ont perçu 38,5 \% (European Commission, 2013).

12. Ce moratoire censé protéger le marché foncier des NEM a pris fin en 2011, 2014 ou 2016, selon les pays.

13. Il s'agit de contrats informels entre les parties prenantes. Un million d'hectares pourrait être concerné.

14. Lors de la privatisation du combinat agricole de Bóly, en 2005, le principal investisseur (Sándor Csányi, président de la banque OTP) a pris le contrôle du capital. La firme agro-industrielle Bóly Zrt a été intégrée 
À son arrivée au pouvoir en 2010, le parti Fidesz qui se présentait comme le protecteur des petits paysans, a tenté de lutter contre ces transactions douteuses en renforçant le code criminel au sujet des « contrats de poche » (juin 2012). Cependant, la nouvelle loi sur la location des terres, adoptée à l'automne 2012, en vue de réguler le marché foncier après la fin du moratoire sur l'achat des terres par les étrangers, a fixé le plafond des baux à 50 ha pour les petits exploitants, à 300 ha pour les exploitations familiales et à 1200 ha pour les structures sociétaires*. Le dispositif législatif révisé se révèle inapte à freiner la concentration foncière et son corollaire, la dépossession des petits propriétaires ${ }^{15}$. L'absence de compétitivité des petites exploitations, le vieillissement démographique de leurs chefs d'exploitation, précipitent leur sortie des circuits marchands, puis l'abandon de la production de subsistance.

En Roumanie comme en Bulgarie, le processus d'accaparement foncier est le fait de structures du type « agroholding* », détenues par des capitaux étrangers (fonds d'investissement européens et internationaux) qui cherchent à diversifier leur portefeuille. Le mouvement d'accaparement a donné naissance à un groupe d'entrepreneurs associant intermédiaires et spéculateurs (voire arnaqueurs), qui se portent acquéreurs au terme de transactions douteuses.

Qu'elles soient roumaines ou étrangères, les firmes* qui s'accaparent les terres s'allient aux autorités pour orienter la législation et les programmes de développement locaux et nationaux en leur faveur. Elles exploitent la vulnérabilité de la population et la faiblesse des institutions pour prendre le contrôle des terres, à travers l'achat de parcelles ou par des contrats de location abusifs ${ }^{16}$.

\section{Conclusion}

Le caractère inédit des transformations agraires intervenues dans les pays sortis du système collectiviste, invite à penser en termes nouveaux les héritages structurels. Dans une histoire marquée par une longue série de réformes foncières, au cours du $\mathrm{Xx}^{\mathrm{e}}$ siècle, la décollectivisation* peut être vue comme un nouveau tournant agraire. Mis en œuvre de manière radicale, le changement institutionnel qui procède du rétablissement de la propriété privée du sol et du passage à l'économie de marché, a provoqué un enchaînement de mutations qui ont remodelé les structures d'exploitation de la terre. Loin d'effacer les héritages structurels, les trajectoires agraires s'inscrivent dans la durée du temps historique, renouant avec les legs du passé pour infléchir les choix orientant les itinéraires de sortie du système collectiviste. Les formes sociales de production observables dans les agricultures post-collectivistes sont le produit de ces trajectoires sociohistoriques.

Le changement institutionnel a ouvert un champ d'opportunités aux acteurs économiques. Sous des formes juridiques diverses, la privatisation de la terre et du capital a permis la recomposition des facteurs de production, en convertissant les organisations préexistantes ou en créant de nouvelles formes sociales de production. La reconstitution de la propriété privée du sol ne s'est pas accompagnée d'un mouvement de

à la holding Bonafarm, premier groupe agroalimentaire du pays, qui détient le capital de quatre entreprises agro-industrielles et de trois usines de transformation.

15. Selon Jozsef Angyan, ancien secrétaire d'État au ministère de l'Agriculture : «Des réseaux mafieux s'accaparent la totalité des ressources, de la terre, des subventions et des marchés » (La France agricole, 2013).

16. Voir les études de terrain effectuées sous la direction de Judith Bouniol pour Ecoruralis (2013, p. 5).

(C) Presses universitaires de Franche-Comté http://pufc.univ-fcomte.fr/ 
réappropriationindividuelledelaterre, ainsiquedesmoyensdeproduction, demêmeampleur. De la désarticulation entre terre, capital et travail sont nés de nouveaux rapports sociaux en agriculture. Parce qu'elle procède d'une adaptation à des changements qui font intervenir des institutions informelles et formelles évoluant à leur rythme propre, la recomposition a emprunté des voies plurielles ménageant des formes transitoires, telles que, par exemple, les coopératives ou les associations de propriétaires. La période de transition s'est étendue sur toute la décennie 1990-2000. La perspective de l'intégration dans l'UE est venue modifier la donne institutionnelle, en déclenchant une nouvelle série d'adaptations des structures d'exploitation.

Quelques tendances majeures se dessinent:

- La reconstitution d'une agriculture de type familial n'a pas été la voie majoritaire empruntée par les pays d'Europe centrale ayant subi une collectivisation* intégrale. La perte des compétences et des savoir-faire propres à la paysannerie, le manque de capitaux et de réseaux d'encadrement technique, l'absence d'organisations professionnelles, ont contribué à freiner la formation d'un modèle familial moderne et durable. Dans ces conditions, le référentiel de l'agriculture familiale*, entendue comme «le modèle opérationnel dominant en Europe», s'est révélé inadapté aux formes d'organisation de la plupart des agricultures postcollectivistes et impropre à soutenir la consolidation des petites exploitations des ménages ruraux.

- Le processus de concentration au profit des grandes exploitations progresse aux dépens de toutes les autres catégories. Les mécanismes de dépendance du chemin suivi* ont joué en faveur de la continuité des très grandes exploitations de forme sociétaire*. La grande maille agraire, élément invariant de la trame d'usage du sol, a imposé une nouvelle fois sa logique. Le processus de déconcentration* foncière qui avait suivi la conversion des anciennes structures collectives en diverses formes de type coopératif, associatif ou sociétaire, s'est inversé au fur et à mesure que les forces économiques opéraient une sélection fragilisant les initiatives individuelles. Au cours de la décennie écoulée, le processus d'élargissement de l'assise foncière des grandes exploitations a repris avec une ampleur que l'on n'attendait pas, étant donné la situation d'émiettement de la propriété du sol. L'accaparement foncier* au profit des investisseurs contrôlant les agroholdings* est grandement facilité par les caractéristiques d'un marché foncier dont les règles sont aisément contournables lorsqu'elles ne sont pas sciemment modifiées par la puissance publique. À quelques exceptions près, la marque d'un dualisme agraire de longue durée, s’impose à nouveau en Europe centrale.

\section{Bibliographie}

«Adoption de la loi sur les terres agricoles » [en ligne], La France agricole, 24 juin 2013, URL : < http://www.lafranceagricole.fr/actualites/hongrie-adoption-de-la-loisur-les-terres-agricoles-1,0,87453937.html>.

Bazin Gilles et Bourdeau-Lepage Lise, 2011, "L'agriculture dans les pays d'Europe centrale et orientale. Continuité et adaptation », Économie rurale, vol. 5, n 325 326 , p. 10-24. 
BounIoL Judith, 2013 (janvier), L'accaparement des terres en Roumanie, menace pour les territoires ruraux [en ligne], URL : <http://slowfood.com/filemanager/landgrabbing/ecoruralis.pdf $>$.

Davidova Sophia, Bailey Alastair, Dwyer Janet, Erjavec Emil, Gorton Matthew, Thomson Kenneth, 2013, Semi-subsistence farmig: values and directions of development [en ligne], DG for Internal policies, Policy department B : Structural and cohesion policies, European Parliament, URL : <http://www.europarl.europa.eu/RegData/ etudes/etudes/join/2013/495861/IPOL-AGRI_ET(2013)495861_EN.pdf.>

Davidova Sophia et Thomson Kenneth, 2014, Family farming in Europe: challenges and prospects [en ligne], DG for Internal policies, Policy department B: Structural and cohesion policies, European Parliament, URL : <http:// www.europarl.europa.eu/RegData/etudes/note/join/2014/529047/ IPOL-AGRI_NT(2014)529047_EN.pdf>.

Darrot Catherine, Hirschhausen Béatrice (von), 2011, «PAC et transition agricole en Pologne et Roumanie : les nouveaux termes du processus », Économie rurale, vol. 5, $\mathrm{n}^{\circ} 325-326$, p. 69-84.

European Commission, 2013, Report on the distribution of direct aids to agricultural producers (financial year 2013) [en ligne], URL : <https://ec.europa.eu/agriculture/sites/agriculture/files/cap-funding/beneficiaries/direct-aid/pdf/ annex2-2013_en.pdf>.

— 2011 (juillet), "What is a small farm? », EU Agricultural Economics Briefs, $\mathrm{n}^{\circ} 2$ [en ligne], URL : <https://ec.europa.eu/agriculture/sites/agriculture/files/ rural-area-economics/briefs/pdf/02_en.pdf $>$.

European Coordination Via Campesina (ECVC), Hands-Off The Land (HOTL), 2013, Land concentration, land grabbing and people's struggles in Europe [en ligne], Final Report, URL : < https://www.tni.org/files/download/land_in_europe-jun2013. $\operatorname{pdf}>$.

Eurostat, 2013, Agriculture, forestry and fishery statistics. 2013 edition [en ligne], Luxembourg, Publications Office of the EU, URL : < https://ec.europa. eu/eurostat/documents/3930297/5968754/KS-FK-13-001-EN.PDF/ ef39caf7-60b9-4ab3-b9dc-3175b15feaa6>.

Halamska Maria, 2011, "The Polish Countryside in the Process of Transformation 1989-2009 », Polish sociological review, n 173, p. 35-54.

Halamska Maria et Maurel Marie-Claude, 2010. «Decollectivization of Agriculture and Reshaping of Agrarian Structure in Central Europe », Praglad Socjologiczny, vol. $59, \mathrm{n}^{\circ} 2$, p. $29-56$.

Hartvigsen Morten, 2014, " Land reform and land fragmentation in Central and Eastern Europe », Land use policy, n 36, p. 330-341.

HirschHausen Béatrice (von), 1997, Les nouvelles campagnes roumaines. Paradoxes d'un retour paysan, Paris, Belin.

Martins Carla et TosstorfF Guenther, 2011, "Large Farms in Europe ", Statistics in Focus : Agriculture and Fisheries, $\mathrm{n}^{\circ} 18$ [en ligne], URL : < https:// 
ec.europa.eu/eurostat/documents/3433488/5578740/KS-SF-11-018-EN. PDF/4330ef6b-9501-4af5-b4d4-139c04273561>.

Maurel Marie-Claude, 2015, «From disappearance to recovery: Family farming in Central Europe. Questioning the path dependency hypothesis », Wies $i$ Rolnictwo, $\mathrm{n}^{\circ} 1$, p. $79-105$.

— 2012 (juillet-décembre), « La grande maille agraire en Europe centrale : un invariant spatiotemporel ?", Études rurales, n 190 , p. 25-48.

— 1994, La transition post-collectiviste. Mutations agraires en Europe centrale. Paris, L'Harmattan.

Maurel Marie-Claude, Halamska Maria et Lamarche Hugues, 2003, Le repli paysan. Trajectoires de l'après-communisme en Pologne, Paris, L'Harmattan.

Purseigle François (coord.), 2012 (décembre), Études rurales, n 190 Les agricultures de firme. 1) Organisations et financiarisation.

Swain Nigel, 2013, "Agriculture "East of the Elbe" and the Common Agricultural Policy », Sociologia Ruralis, vol. 53, n 3 , p. 369-389.

Treuillaud-Paun Aurélia, 1977 (juillet-septembre), « Le nouveau concept de la propriété en Roumanie », Revue internationale de droit comparé, vol. 49, n 3, p. 575-585.

Vandenbroucke Perrine et FehÉR Istvàn, 2011, « Dynamique des structures agraires hongroises : Quels profils d'exploitations se dessinent? », Économie rurale, n 325 326, p. 100-113. 


\section{Table des matières}

\section{Introduction}

Gérard Chouquer et Marie-Claude Maurel 9

Première partie : Évolutions et révolutions dans les politiques agricoles France

1 Les révolutions agricoles contemporaines en France Sophie Devienne.

2 L'espace politique agricole français (1945-2015)

Clémence Guimont et Bruno Villalba.....

3 Le droit du sol et le sang de la terre Hubert Bosse-Platière

\section{Europe}

4 La politique agricole commune : un compromis européen en crise Aurélie Trouvé et Gilles Bazin.

5 Réappropriation foncière dans les campagnes d'Europe centrale Marie-Claude MAUREL

6 Ressource foncière, rente et mutations des agricultures méditerranéennes Pascal Chevalier et Guillaume LACQuement. 


\section{Seconde partie : Interrogations autour des modèles}

7 Des entreprises agricoles « aux allures de firmes » François Purseigle, Guilhem AnZalone, Geneviève NGuYen et Bertrand Hervieu 163

8 Le modèle de l'agriculture familiale en France Hubert COCHET

9 L'inquiétant modèle du « travail saisonnier » en Europe Gérard Chouquer

10 Interrogations théoriques autour de la propriété et du foncier en Europe Gérard Chouquer

Liste des sigles et acronymes 257

Lexique 261

Index géographique 277

Ont collaboré à l'ouvrage 281

Table des figures.. 289 
Imprimerie Messages

111, rue Nicolas Vauquelin - 31100 Toulouse

Dépôt légal : premier trimestre 2019

Imprimé en France 
L'ouvrage propose un tour d'horizon des évolutions les plus récentes du foncier et de l'agriculture en Europe. Une première partie caractérise les évolutions et les révolutions des politiques agricoles, étudiées du point de vue du droit, de la science politique, de l'agronomie et de la géographie, en mettant l'accent sur la fin d'une phase protectionniste, et avec deux éclairages régionaux, en Méditerranée et dans l'Europe médiane. Une seconde partie interroge les modèles agricoles et fonciers : I'agriculture familiale, l'agriculture de firme, le travail saisonnier, les mutations de la propriété et l'évolution vers le portage foncier.

Gérard CHOUQUER, historien, est directeur de recherches au CNRS honoraire et membre correspondant de l'Académie d'agriculture de France.

Marie-Claude MAUREL, géographe, est directrice d'études à l'EHESS et membre de l'Académie d'agriculture de France.

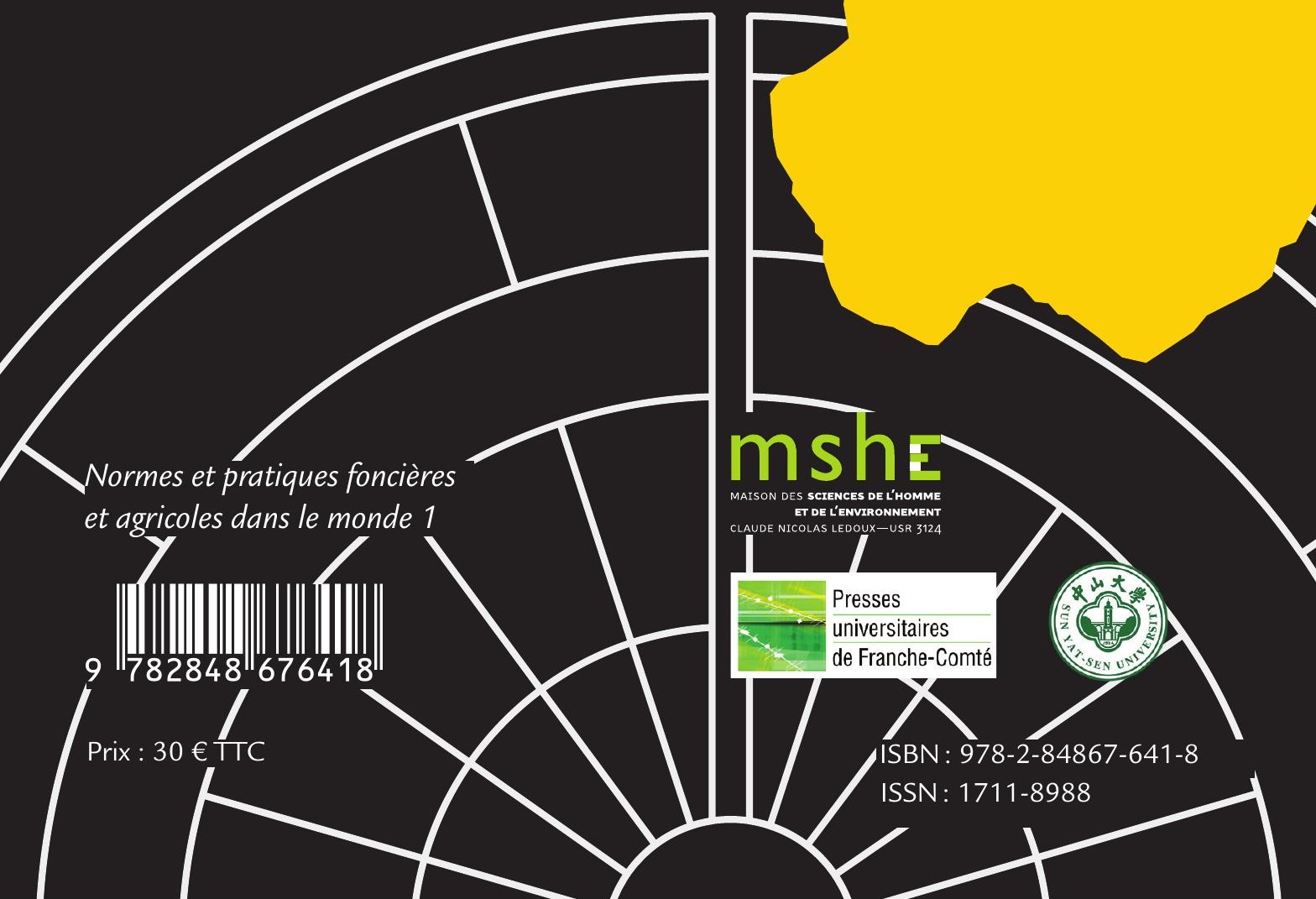

